\title{
How can econometrics help fight the COVID-19 pandemic?
}

\author{
Kevin Alvarez and Vladik Kreinovich \\ Department of Computer Science, University of Texas at El Paso, \\ El Paso, Texas, USA
}

Abstract

Purpose - The current pandemic is difficult to model - and thus difficult to control. In contrast to the previous epidemics, whose dynamics were smooth and well described by the existing models, the statistics of the current pandemic are highly oscillating. The purpose of this paper is to explain these oscillations and to see how this explanation can be used to fight the epidemic.

Design/methodology/approach - The authors use an analogy with economic systems.

Findings - The authors show that these oscillations can be explained if we take into account the disease's long incubation period - as a result of which our control measures are determined by outdated data, showing number of infected people two weeks ago. To better control the pandemic, the authors propose to use the experience of economics, where also the effect of different measures can be observed only after some time. In the past, this led to wild oscillations of the economy, with rapid growth periods followed by devastating crises. In time, economists learned how to smooth the cycles and thus to drastically decrease the corresponding negative effects. The authors hope that this experience can help fight the pandemic.

Originality/value - To the best of our knowledge, this is the first explanation of the highly oscillatory nature of this epidemic's dynamics.

Keywords Econometrics, COVID-19 pandemic, Oscillatory dynamics, Time-delay systems

Paper type Research paper

\section{Formulation of the problem}

Need for a model. To properly control the pandemic, it is important to be able to predict the effect of different possible measures. For this purpose, we need a model that would predict how the number of infected and recovered people will change if we implement different measures.

The existing models do not work well. There are many mathematical models describing the spread of epidemics - and the effect of different measures on this spread. However, these models do not describe the current pandemic very well: indeed, these models usually predict smooth changes (Bjornstad, 2018; Brauer et al., 2018; Britton and Pardoux, 2019;

(C) Vladik Kreinovich and Kevin Alvarez. Published in Asian Journal of Economics and Banking. Published by Emerald Publishing Limited. This article is published under the Creative Commons Attribution (CC BY 4.0) licence. Anyone may reproduce, distribute, translate and create derivative works of this article (for both commercial and non-commercial purposes), subject to full attribution to the original publication and authors. The full terms of this licence may be seen at http:// creativecommons.org/licences/by/4.0/legalcode

JEL classification - A12, C50, E17, E32, Y80

MSC2020 classification - 92C60, 92D30, 37N25

This work was supported in part by the National Science Foundation grants 1623190 (A Model of Change for Preparing a New Generation for Professional Practice in Computer Science) and HRD1242122 (Cyber-ShARE Center of Excellence).

The authors are thankful to Dr. Matt Briggs for valuable suggestions. 
AJEB

4,3

Martcheva, 2015; Yan and Chowell, 2019), while for the current pandemic, the changes are heavily oscillating, even after we try to smooth this data by taking five-day averages instead of daily data; see, e.g. Figures 1 and 2.

Oscillations in the first picture may be partly explained by the uneven progress of testing, but the second picture, describing the number of hospitalized people, shows similar oscillations as well.

What can we do? In this paper, we provide a possible explanation for this oscillation, and we explain how the experience of econometrics can help.

\section{Oscillations: possible explanation}

Possible explanation: general idea. What distinguishes the current pandemic from all previous epidemics is the unusually long incubation period. It can take up to two weeks before the infected person shows any symptoms of the disease.

As a result, when we make a decision - e.g. to enforce or ease restrictions - we do not know the current number of infected people, we only know the number of sick people which is proportional to the number of infected people two weeks ago. In other words, when we impose some restrictions - whether it is on the level of a city or on a level of individual family decisions - our decisions are based on outdated data.

How this explains oscillations: a quantitative explanation. Once the severity of a situation becomes known, we impose several restrictions trying to cut down the number of new infections. If the number of newly sick people does not go down - or even continue to grow more and more severe restrictions are placed, until the numbers start going down.

Once the numbers go down, a natural idea is to ease the restrictions, all the way to completely eliminating them. As the virus is still there, this inevitably leads to some people getting infected. If we had a good idea of how many new people get infected, we could

Figure 1. Typical COVID-19 statistics

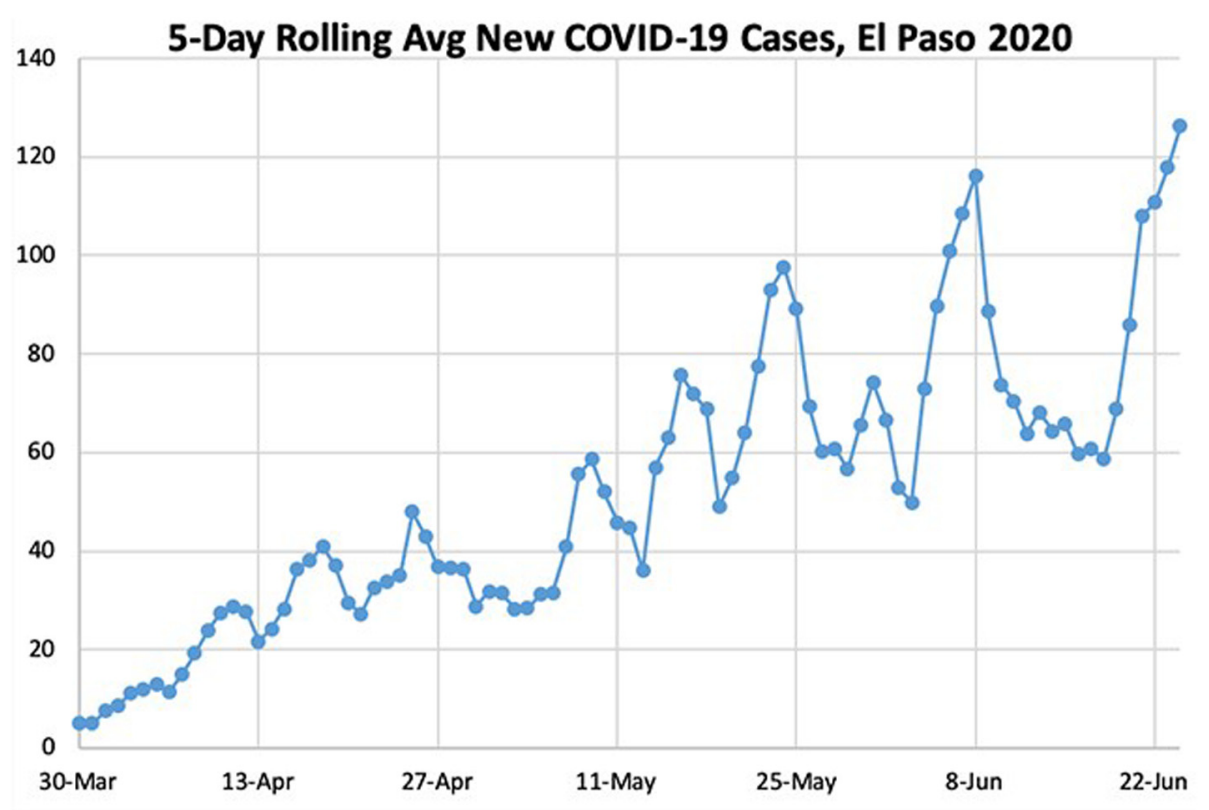




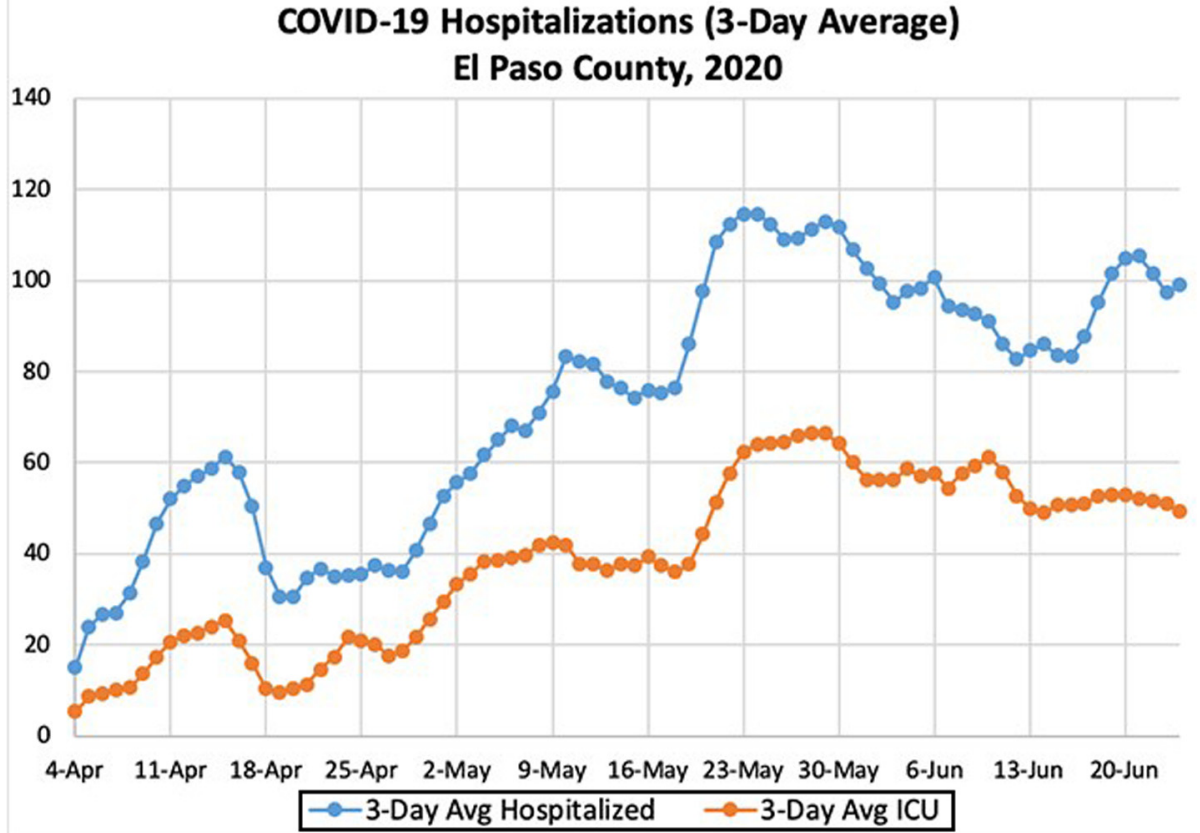

implement appropriate measures and slow down the spread of the pandemic. However, we do not have that information, we only have the information about the number of infected people two weeks ago - when this number was small. So, for two following weeks, we are under the false impression that the situation has improved, while in reality, in the absence of needed restrictions, the epidemic grows exponentially - and we only impose restrictions two weeks later, when the epidemic has already grown a lot. So, more severe restrictions are reintroduced, etc. - and this cycle repeats again and again.

A simple quantitative model explaining such oscillations. Let us introduce a simple quantitative model that explains such oscillations.

In the absence of any restrictions, each infected person infects, on average, a certain number $r>1$ of new people during a certain period of time (e.g. a week). So, the number $x(t+1)$ of people newly infected during the next period of time is proportional to the previous number $x(\mathrm{t})$, with the coefficient of proportionality $r$.

$$
x(t+1)=r \cdot x(t) .
$$

In this case, we have the exponential growth typical for the starting period of each epidemic: because of equation (1):

- for $t=0$, we get $x(1)=r \cdot x(0)$;

- for $t=1$, we get $x(2)=r \cdot x(1)$; substituting the above expression for $x(1)$ into this formula, we get $x(2)=r \cdot(r \cdot x(0))=r^{2} \cdot x(0)$; and

- for $t=2$, we get $x(3)=r \cdot x(2)$; substituting the above expression for $x(2)$ into this formula, we get $x(3)=r \cdot\left(r^{2} \cdot x(0)\right)=r^{3} \cdot x(0)$.

In general, we get $x(t)=r^{t} \cdot x(0)$.
COVID-19

pandemic

Figure 2.

Typical COVID-19 statistics (continued) 
AJEB

4,3

What if we impose restrictions? The restrictions decrease the number of newly infected people. The severity of restrictions - and thus, their effect - is proportional to the observed data, and the observed data reflects the number of newly infected people two week ago, i.e. the value $x\left(t-t_{0}\right)$, where $t_{0}$ is the incubation period (in our case, two weeks). Let us denote the proportionality coefficient by $k$. Then, in the first approximation, we can replace equation (1) with the following one:

$$
x(t+1)=r \cdot x(t)-k \cdot x\left(t-t_{0}\right) .
$$

We said that this is only the first approximation, as two important corrections are needed.

First, if the number of new cases is very small, i.e. smaller than some small threshold $x_{0}$, then no restrictions will be imposed. So, the effect of restrictions should be proportional not to the actual value $x\left(t-t_{0}\right)$, but rather to the excess of this value in comparison with $x_{0}$, i.e. to $\left.\max \left(x\left(t-t_{0}\right)-x_{0}\right), 0\right)$ Thus, the equation takes the following form:

$$
x(t+1)=r \cdot x(t)-k \cdot \max \left(x\left(t-t_{0}\right)-x_{0}, 0\right) .
$$

Second, no matter how severe are the restrictions, we cannot fully eliminate the virus, so the number $x(t+1)$ cannot go below the small value $x_{0}$. In other words, if the right-hand side of equation (3) is smaller than $x_{0}$, the new value $x(t+1)$ will still be equal to $x_{0}$. So, instead of the right-hand side of equation (3), we should take the largest of this right-hand side and $x_{0}$. Thus, we arrive at the final equation describing our model:

$$
x(t+1)=\max \left(x_{0}, r \cdot x(t)-k \cdot \max \left(x\left(t-t_{0}\right)-x_{0}, 0\right)\right) .
$$

This equation describes the effect of control when we have already realized that there are infected people in the community: we will do that after time $t_{0}$, when the first infected people get sick.

So, for $t \geq t_{0}$, we have equation (4), while for $t<t_{0}$, we use the uncontrolled equation (1).

This model indeed leads to oscillations. Let us show, on a simple example, that the above equations (1) - (4) indeed leads to oscillations.

In this example, we start with:

- the simplest number infected persons - one: $x(0)=1$;

- the simplest value $x_{0}>1: x_{0}=2$;

- the simplest value $r$ for which the infection grows if not controlled: $r=2$;

- the simplest value $k>r: k=3$; we need to have $k>r$ to make sure that the control is effective; and

- the actual incubation period $t_{0}=2$ weeks.

Then, we have the following dynamics:

- at first:

$$
x(0)=1
$$

- to get the value $x(1)$, we use equation (1) with $t=0$, getting:

$$
x(1)=2 \cdot x(0)=2 \text {; }
$$

- to get the value $x(2)$, we use equation (1) with $t=1$, getting:

$$
x(2)=2 \cdot x(1)=4
$$


- to get $x$ (3), we already need to use equation (4), which leads to:

$$
x(3)=\max (2,2 \cdot 4-3 \cdot 0)=\max (2,8)=8 ;
$$

- $\quad$ for $x(4)$, we get

$$
x(4)=\max (2,2 \cdot 8-3 \cdot 0)=16
$$

- for $x(5)$, we get:

$$
x(5)=\max (2,2 \cdot 16-3 \cdot(4-2))=\max (2,32-6)=26 ;
$$

- for $x(6)$, we get:

$$
x(6)=\max (2,2 \cdot 26-3 \cdot(8-2))=\max (2,52-18)=34 ;
$$

- for $x(7)$, we get:

$$
x(7)=\max (2,2 \cdot 34-3 \cdot(16-2))=\max (2,68-42)=26 ;
$$

finally, the number of new cases started decreasing;

- for $x(8)$, we get:

$$
x(8)=\max (2,2 \cdot 26-3 \cdot(26-2))=\max (2,52-72)=2 ;
$$

we think we won;

- for $x(9)$, we get:

$$
x(9)=\max (2,2 \cdot 2-3 \cdot(34-2))=2 ;
$$

- for $x(10)$, we get:

$$
x(10)=\max (2,2 \cdot 2-3 \cdot(26-2))=2 ;
$$

- $\quad$ for $x(11)$, we get:

$$
x(11)=\max (2,2 \cdot 2)=4
$$

the number of infected people starts growing again, but we do not know it yet;

- $\quad$ for $x(12)$, we get:

$$
x(12)=\max (2,2 \cdot 4)=8
$$

- $\quad$ for $x(13)$, we get:

$$
x(13)=\max (2,2 \cdot 8)=16
$$

- for $x(14)$, we get:

$$
x(14)=\max (2,2 \cdot 16-3 \cdot(4-2))=\max (2,32-6)=26 ;
$$

- finally, we notice the growth and start taking measures.

Then, the loop repeats again: we get $x(15)=x(6)=34, x(16)=x(7)=26, x(17)=x(8)=2$, and we get oscillations with period $T=9$. 
AJEB

4,3

\section{How econometrics can help: idea}

\subsection{General idea}

The current pandemic is not the only case when we can only see the delayed effect of each measure. Such situations are typical in economics. Whatever measures we implement in economics, be it a change in taxes of tariffs, a change in the interest rate, etc. - these measures do not immediately affect the gross domestic product (GDP), unemployment level or any other important economic characteristic, the effect is usually delayed.

The delayed character of economics is, in our opinion, one of the main reasons for the economic cycles. These cycles are easy to explain, e.g. if one year, there is a lot of demand for some agriculture crop, its price goes up, so next year, everyone switches to this crop, and the resulting production becomes much larger than the demand. So, the price goes down, and many farmers lose money.

In the past, the oscillations were wild, and the cycles were really devastating, with huge unemployment and low GDP during the crisis period. At present, economists learned to smooth these cycles - i.e. to control the corresponding delayed systems (Greene, 2018; Hurn et al., 2020; Wooldridge, 2019).

We hope that this experience will help control the current epidemic as well.

\subsection{Why use experience of economies and not of some other time-delay systems}

Economies are not the only systems in which the effect of a control measure appear only with some delay. There are other systems of this type, e.g. in engineering, in ecology, etc. (Fridman, 2014; Park et al., 2019). In this paper, however, we concentrate on the experience of economic systems: while engineering time-delay systems are somewhat exotic and not yet well studied, economy is what we all understand, what we all experience, in which there is a lot of experience of successful and unsuccessful control.

\subsection{What exactly we can learn from economics}

One of the ideas that economists have learned - and that the econometric models confirm - is that we need to keep some control of the economy even when this economy sounds healthy.

One of the main reasons why in the past economy regularly went through disastrous crisis cycles is that control was only applied when it became clear that the situation has become critical - by which time it was too late (because of the delay effect) to prevent the crisis from happening. Now, we are smarter. Even when the economy is growing and all the signs are good, we know that there is a possibility of "overheating" that could potentially lead to a crisis. So, we impose restrictions, slowing down the growth - and thus either avoid the crisis altogether or at least make the effects of the crisis less painful.

Thus, a natural idea is to use the same approach to fighting the epidemic: instead of completely easing restrictions when the situation become good, we need to keep some restrictions - this will hopefully help us fight the epidemic.

Let us describe this idea in some detail.

\section{How econometrics can help: details}

\subsection{Main idea: in detail}

In the previous section, we have shown that if we control the epidemic based only on the current number of sick people - which reflects the number of infected people two weeks ago - we are bound to have oscillations instead of the desired end to the epidemic:

- at first, we cut down on the number of new cases; then

- we ease the restrictions and the number of infected people starts to rise again. 
Once we have reached the long period of low number of newly sick folks, if we completely eliminate the restrictions, we may have a new outburst - that we will not notice for two weeks, during which time, it may again become catastrophic. So, to avoid the new outburst, we need to keep some restrictions, even when the number of cases stays very low.

\subsection{How to formalize this idea}

In the previous section, we assumed that the control is proportional to the excess of the number of new cases over the minimum $x_{0}$ - so that when this number becomes equal to $x_{0}$ or smaller than $x_{0}$, we do not apply any control.

Instead, we need to keep the pressure going even in this case. So, in addition to the control $k \cdot \max \left(x\left(t-t_{0}\right)-x_{0}, 0\right)$, which is proportional to the difference:

$$
x\left(t-t_{0}\right)-x_{0},
$$

we should also apply an additional constant control $c$, for some appropriate constant $c$ selected so as to prevent the outburst.

In this case, instead of equation (4), we have a formula:

$$
x(t+1)=\max \left(x_{0}, r \cdot x(t)-\left(k \cdot \max \left(x\left(t-t_{0}\right)-x_{0}, 0\right)+c\right)\right) .
$$

\subsection{This indeed helps}

In the above numerical example, to avoid the second outburst, it sufficient to take $c=2$. If we apply this additional control from the very beginning, then, when we start with $x(0)=1$, we will get the following:

- first, we get

$$
x(1)=\max (2,2 \cdot 1-2)=2
$$

- $\quad$ after that, we get:

$$
x(2)=\max (2,2 \cdot 2-2)=2 ;
$$

and after that we have $x(3)=x(4)=\ldots=2$ : no outburst.

We get the exact same result is we start applying the additional control value $c$ not from the very beginning, but after some time. For example, in the above simulation, if we apply this additional control $c=2$ at moment $t=10$, at which the number of newly sick people $x\left(t-t_{0}\right)$ becomes smaller than or equal to the threshold, we will avoid the repetition of the loop. Indeed, in this case, we will have:

- $\quad$ for $x(11)$, we get:

$$
x(11)=\max (2,2 \cdot 2-2)=2
$$

- for $x(12)$, we similarly get:

$$
x(12)=\max (2,2 \cdot 2-2)=2
$$

and so on, the numbers never increase above the desired threshold $x_{0}$. 
AJEB

4,3

36

\subsection{Important comment}

As we have mentioned earlier:

- an important part of the control comes from the officially introduced measures, like wearing masks and keeping social distance;

- however, a significant part of the control comes from people following these measures, and, in general, exercising caution.

It is easy to impose measures of different levels of severity, but it is difficult to control people's behavior. No matter what measures we impose, if people see that for some period of time, the number of new cases remains low, they will ease their self-control. To compensate for that, we need to impose stricter official measures.

This sounds counter-intuitive: we impose stricter measures when the situation becomes better. However, this is the only way to keep the overall pressure constant and thus to avoid the new increase in the number of cases.

\section{References}

Bjornstad, O.N. (2018), Epidemics: Models and Data Using R, Springer, Cham.

Brauer, F., Castillo-Chavez, C. and Feng, Z. (2018), Mathematical Models in Epidemiology, Springer, New York, NY.

Britton, T. and Pardoux, E. (Eds), (2019), Stochastic Epidemic Models with Inference, Springer, Cham.

Fridman, E. (2014), Introduction to Time-Delay Systems: Analysis and Control, Birkhauser, Cham.

Greene, W.H. (2018), Econometric Analysis, Pearson, New York, NY.

Hurn, S., Martin, V.L., Phillips, P.C.B. and Yu, J. (2020), Financial Econometric Modeling, Oxford University Press, Oxford.

Martcheva, M. (2015), An Introduction to Mathematical Epidemiology, Springer, New York, NY.

Park, J.H., Lee, T.H., Liu, Y. and Chen, J. (2019), Dynamic Systems with Time Delays: Stability and Control, Springer, Singapore.

Wooldridge, J.M. (2019), Introductory Econometrics: A Modern Approach, Cengage Learning, Boston.

Yan, P. and Chowell, G. (2019), Quantitative Methods for Investigating Infectious Disease Outbreaks, Springer, Cham.

Corresponding author

Vladik Kreinovich can be contacted at: vladik@utep.edu

For instructions on how to order reprints of this article, please visit our website:

www.emeraldgrouppublishing.com/licensing/reprints.htm

Or contact us for further details: permissions@emeraldinsight.com 